\title{
Associação de comorbidades e hábitos não saudáveis com a capacidade de caminhada em pacientes com claudicação intermitente
}

CDD. 20.ed. 613.7

616.1

\author{
J oão Paulo dos Anjos Souza BARBOSA* \\ Mariana de Freitas BERENGUER* \\ Lausanne Barreto de Carvalho Cahú RODRIGUES* \\ Alessandra de Souza MIRANDA* \\ Luis Alberto GOBBO** \\ Gabriel Grizzo CUCATO*** \\ Cláudia Lúcia de Moraes FORJ AZ ${ }^{\text {** }}$ \\ Maria de Fátima Nunes MARUCCI** \\ Raphael Mendes RITTI-DIAS* \\ *Escola Superior de \\ Educação Física, Uni- \\ versidade de Pernam- \\ buco. \\ **Faculdade de Saúde \\ Pública, Universidade \\ de São Paulo. \\ ***Escola de Educa- \\ ção Física e Esporte, \\ Universidade de São \\ Paulo.
}

\section{Resumo}

Indivíduos com claudicação intermitente $(\mathrm{Cl})$ comumente apresentam comorbidades associadas e hábitos não saudáveis, como o tabagismo e a inatividade física. Todavia, o quanto esses fatores contribuem para a intolerância à caminhada desses individuos permanece incerto. Assim, o objetivo deste estudo foi analisar a associação das comorbidades e hábitos não saudáveis com a capacidade de caminhada de indivíduos com $\mathrm{Cl}$. A amostra foi composta por 66 indivíduos com $\mathrm{Cl}$ de ambos os gêneros. Todos os indivíduos realizaram teste ergométrico máximo em esteira, sendo registrada o tempo de claudicação (TC) e o tempo total de caminhada (TTC). Os dados referentes às comorbidades e hábitos não saudáveis foram obtidos por meio de entrevista. Para análise estatística foi empregada análise de regressão simples univariada, com $p<0,05$. A comorbidade mais prevalente foi a hipertensão arterial (86,4\%), ao passo que a inatividade física foi o hábito não saudável mais prevalente (47\%). Além disso, 90,9\% dos pacientes apresentaram duas ou mais comorbidades e/ou hábitos não saudáveis. A inatividade física foi associada com menor TC ( $\beta=-154 \mathrm{~s} ; p=0,02 ;$ IC95\% $=-283 ;-25 \mathrm{~s})$ e TTC ( $\beta=-189 \mathrm{~s} ; p=0,02 ;$ IC95\% $=-384 ;-29 \mathrm{~s}$ ). Além disso, o maior número de comorbidades e hábitos não saudáveis também foram associados com menor TC ( $\beta=-55 \mathrm{~s} ; \mathrm{p}=0,02 ;$ IC95\% $=-102 ;-8 \mathrm{~s})$. Como conclusão, os resultados deste estudo indicam que a inatividade física e o número de comorbidades e/ou hábitos não saudáveis estão associados com a intolerância à caminhada de indivíduos com $\mathrm{Cl}$.

UnIteRMos: Doenças, fatores de risco, Doença Arterial Obstrutiva Periférica.

\section{Introdução}

A doença arterial obstrutiva periférica (DAOP) é causada por lesōes ateroscleróticas que diminuem o fluxo sanguíneo nos tecidos distais à estenose arterial, sendo mais comumente observada nos membros inferiores (MrTChelL, 2008). A claudicação intermitente $(\mathrm{CI})$, caracterizada por dor, câimbra, ardência ou formigamento que ocorre durante a prática de atividade física, é o principal sintoma da
DAOP (Hirsch, Haskal, Hertzer, Bakal, Creager, Halperin, Hiratzka, Murphy, Olin, Puschett, Rosenfield, Sacks, STanley, Taylor, White, White, White, Antman, Smith, Adams, Anderson, FAXon, Fuster, GibBons, Hunt, Jacobs, Nishimura, Ornato, Page \& Riegel, 2006). Dessa forma, a CI tem repercussão importante na vida do indivíduo, pois pode limitar a realização de atividades físicas 
cotidianas (Gardner, Montgomery, Scott, AfaQ \& BLEVINS, 2007), gerando redução da aptidão física (Herman, Liu, Tian, Guralnik, Ferrucci, Criqui, Liao \& McDermott, 2009; Kuo \& Yu, 2008) e da qualidade de vida dos indivíduos (SPRONK, BosCH, Veen, Den Hoed \& HuninK, 2005).

No Brasil, a DAOP atinge quase $10,5 \%$ da população geral sendo mais prevalente em indivíduos idosos, alcançando uma prevalência de 21,6\% em indivíduos com mais de 60 anos (Makdisse, PereIra, Brasil, Borges, Machado-Coelho, Krieger, Nascimento Neto \& Chagas, 2008). Dessa forma, a DAOP geralmente ocorre em indivíduos que já apresentam outras doenças crônicas estabelecidas (MAKDisse et al., 2008), tais como hipertensão arterial (GARG, Tian, Criqui, Liu, FerrucCi, GURALNIK, TAN \& McDermotT, 2006), diabetes melitos (Marubito, D’Agostino, Silbershatz \& Wilson, 1997), dislipidemias (Hasimu, LI, NAKAYAMA, YU, YANG, LI \& Hu, 2006), obesidade (MLACAK, BLINC,

\section{Métodos}

\section{Amostra}

Estudo descritivo, transversal, foi realizado com 66 pacientes com DAOP e sintomas de CI, com idade entre 50 e 85 anos, de ambos os gêneros, que foram recrutados do ambulatório do Hospital das Clínicas da Universidade de São Paulo. Os pacientes foram recrutados para a participação em estudo maior, que envolvia a realização de treinamento físico supervisionado, e os dados apresentados são oriundos da avaliaçãoo pré-participação. Foram incluídos no estudo os indivíduos que apresentavam: grau II de DAOP (Fontaine, Kim \& Kieny, 1954), sintomas de CI há mais de seis meses, sintomas de CI durante o teste ergométrico, índice tornozelo braço (ITB) $<0,90$, diminuição do ITB após exercício, conseguiam caminhar, no mínimo, dois minutos com velocidade de $3,2 \mathrm{~km} / \mathrm{h}$. Foram excluídos do estudo, os indivíduos que tinham sido submetidos à cirurgia de revascularização ou angioplastia há menos de um ano e que conseguiam caminhar mais de 20 minutos ininterruptamente no teste ergométrico.

Todos os indivíduos foram devidamente esclarecidos sobre os objetivos e procedimentos que seriam realizados no estudo e, posteriormente, aqueles que concordaram em participar, assinaram o termo de consentimento livre e esclarecido. Este estudo foi
Pohar \& STARe, 2006) e cardiopatias (Hasimu et al., 2006). Além disso, indivíduos com DAOP comumente apresentam hábitos não saudáveis, sendo que os mais prevalentes são o tabagismo (BREVETTI, Silvestro, Schiano \& Chiariello, 2003) e a inatividade física (GARDNER et al., 2007).

Está bem estabelecido na literatura que a presença de comorbidades e hábitos não saudáveis são fatores de risco para a ocorrência da DAOP (HIRSCH et al., 2006). Todavia, o quanto esses fatores contribuem para a intolerância à caminhada nos indivíduos com a doença já estabelecida permanece incerto. $\mathrm{O}$ conhecimento dos fatores relacionados às limitações de locomoção dos indivíduos com CI é de particular importância, pois pode fornecer subsídios teóricos para a elaboração de estratégias que permitam melhorar a capacidade de caminhada dos indivíduos.

Assim, o objetivo deste estudo foi analisar a associação das comorbidades e dos hábitos não saudáveis com a capacidade de caminhada de indivíduos com CI.

aprovado pelo Comitê de Ética em Pesquisa com Seres Humanos da Faculdade de Saúde Pública USP, processo 1370 em 14 de dezembro de 2005.

\section{Teste ergométrico em esteira}

Para quantificação da capacidade de caminhada foi realizado teste ergométrico máximo, em esteira (Inbrasport, modelo ATL, Brasil). Para tanto, utilizou-se o protocolo escalonado específico para indivíduos com CI, com velocidade constante de $3,2 \mathrm{~km} / \mathrm{h}$ e incrementos de dois graus de inclinação a cada dois minutos até a exaustão (GARDNER, Skinner, Cantwell \& Smith, 1991). Todos os indivíduos eram familiarizados previamente com o protocolo de teste. $\mathrm{O}$ teste foi realizado em sala silenciosa, com temperatura mantida entre $20^{\circ}$ e $23^{\circ}$. O teste era interrompido quando os indivíduos não conseguiam mais continuar a caminhada devido à dor nos membros inferiores. Durante o teste foram obtidos os dados referentes ao momento em que o paciente relatou o início do sintoma de dor, caracterizado como tempo de claudicação (TC) e a distância máxima que o paciente conseguiu caminhar mesmo com este sintoma, denominada como tempo total de caminhada (TTC). Estudo anterior indicou que esses procedimentos resultaram em 
coeficiente de reprodutibilidade (r) de 0,89 para a TC e de 0,93 para TTC (GARDNER et al., 1991).

\section{Índice tornozelo braço}

As pressões arteriais sistólica do braço e do tornozelo foram obtidas por meio do método auscultatório usando um esfigmomanômetro de coluna de mercúrio e um "doppler" vascular (Martec, DV 6000, Brasil), e um estetoscópio para as medidas do tornozelo e do braço, respectivamente, seguindo recomendaçôes da literatura (Norgren, HiatT, Dormandy, NeHLER, HARris, FowKes \& RuTHERFord, 2007). As medidas foram obtidas depois de os pacientes terem descansado por 20 minutos na posição supina. O ITB foi calculado para cada perna como a razão da pressão arterial do tornozelo pela do braço, e o menor ITB entre os dois membros foi utilizado.

\section{Variáveis antropométricas}

A massa corporal e a estatura foram obtidas utilizando balança mecânica com estadiômetro acoplado (Welmy, modelo 110, Brasil). As medidas de massa corporal e estatura foram realizadas conforme procedimentos descritos previamente (Gordon, Chumlea \& Roche, 1988). A massa corporal foi medida com precisão de 100 gramas e a estatura com precisão de um centímetro. A partir dos valores de massa corporal e estatura, calculou-se o índice de massa corporal (IMC) por meio da divisão de massa corporal $(\mathrm{kg})$ pela estatura (m), ao quadrado, para o diagnóstico de obesidade.

\section{Comorbidades e hábitos não saudáveis}

Para a obtenção dos dados referentes às comorbidades e os hábitos não saudáveis foi realizada entrevista. Previamente à entrevista os pacientes foram orientados a trazerem todos os exames e receitas de remédios que possuíam. Assim, nessa entrevista os pacientes foram questionados quanto ao seu nível de atividade física, hábitos de tabagismo e doenças associadas utilizando um questionário semi-estruturado.

A hipertensão arterial foi identificada por diagnóstico prévio, uso atual de medicamento anti-hipertensivo, e/ou valores de pressão sanguínea sistólica $\geq 140$ $\mathrm{mmHg}$ e/ou pressão sanguínea diastólica $\geq 90 \mathrm{mmHg}$ (Sociedade Brasileira de Hipertensão. Sociedade Brasileira de Cardiologia. Sociedade Brasileira de Nefrologia, 2004). O diabetes melitos foi identificada por diagnóstico prévio, uso atual de medicamento de diabetes, e/ou glicemia de jejum $\geq 126 \mathrm{mg} / \mathrm{dl}$ (AMERICan Diabetes Association, 1997). A dislipidemia foi identificada por diagnóstico prévio, ou uso de medicamento para controle de triglicerídeos, colesterol total, HDL, LDL, e VLDL. A obesidade foi identificada por valores de IMC $\geq 30,0 \mathrm{~kg} / \mathrm{m}^{2}$. A presença de doença cardíaca foi determinada por história anterior de doença arterial coronária, angina, e/ou histórico de angioplastia coronária ou revascularização. Foram classificados como inativos fisicamente os indivíduos que realizavam menos que 150 minutos/semana de atividade física de lazer ou transporte. Foram classificados como tabagistas os indivíduos que relataram uso ocasional ou diário de cigarro, charutos ou cachimbos nos últimos seis meses.

\section{Análise estatística}

Foi empregada estatística descritiva incluindo média, desvio padrão e distribuição de frequência. A regressão linear simples foi usada para identificar as comorbidades e os hábitos não saudáveis relacionadas independentemente com a capacidade de caminhada. Um modelo de regressão separado foi realizado para a TC e para a TTC. Em todas as análises um nível de significância de $\mathrm{p}<0,05$ foi adotado.

\section{Resultados}

A maioria da amostra foi composta por homens ( $\mathrm{n}=43 ; 65,7 \%)$, com idade variando de 50 a 85 anos (TABELA 1). A prevalência de comorbidades variou de $24,2 \%$ (obesidade) a 86,4\% (hipertensão arterial). Entre os hábitos não saudáveis, a inatividade física foi a mais prevalente $(47,0 \%)$. Considerando o número de comorbidades e/ou hábitos não saudáveis nenhum paciente apresentou menos de um fator. Além disso, a grande a maioria dos pacientes
(90,9\%) apresentou duas ou mais comorbidades e/ ou hábitos não saudáveis (TABELA 2).

Utilizando procedimentos de regressão linear simples (TABELA 3), a inatividade física ( $\mathrm{p}=$ 0,02) e o número de comorbidades e/ou hábitos não saudáveis $(\mathrm{p}=0,02)$ foram significativamente associados com menor TC. Além disso, a inatividade física também foi associada com menor TTC ( $\mathrm{p}$ $=0,02)$. Todavia, o diabetes melitos $(p=0,06)$, 
BARBOSA, J.P.A.S. et al.

as doenças cardíacas ( $\mathrm{p}=0,09)$ e o número de 0,07 ) apresentaram tendência de associação com comorbidades e / ou hábitos não saudáveis $(\mathrm{p}=$ menor TC ou TTC.

TABELA 1 - Características dos sujeitos da amostra $(\mathrm{n}=66)$.

\begin{tabular}{lc}
\hline \multicolumn{2}{c}{ Variáveis } \\
\hline Percentual de homens $(\%)$ & 65,7 \\
Idade (anos)* & $65,8 \pm 8,4$ \\
Massa Corporal $(\mathrm{kg})^{*}$ & $71,1 \pm 13,7$ \\
Estatura $(\mathrm{m})^{*}$ & $1,61 \pm 0,08$ \\
Índice de Massa Corporal $\left(\mathrm{kg} / \mathrm{m}^{2}\right)^{*}$ & $27,3 \pm 4,6$ \\
Índice tornozelo braço* & $0,55 \pm 0,10$ \\
Tempo de claudicação $(\mathrm{s})^{*}$ & $411 \pm 270$ \\
Tempo total de marcha $(\mathrm{s})^{*}$ & $706 \pm 336$ \\
\hline
\end{tabular}

TABELA 2 - Prevalência dos fatores de risco de indivíduos com claudicação intermitente (n=66).

\begin{tabular}{lccc}
\hline \multicolumn{1}{c}{ Variáveis } & N & \% \\
\hline Inatividade Física & 31 & 47,0 \\
Tabagismo & 17 & 25,8 \\
Hipertensão arterial & 57 & 86,4 \\
Diabetes melitos & 31 & 47,7 \\
Obesidade & 16 & 24,2 \\
Dislipidemia & 37 & 57,8 \\
Cardiopatias & 25 & 37,9 \\
Número de comorbidades e/ou hábitos não saudáveis & & & \\
& 1 & 6 & 9,1 \\
& 2 & 17 & 25,8 \\
& 3 & 15 & 22,7 \\
& 4 & 15 & 22,7 \\
& 5 & 9 & 13,6 \\
\hline
\end{tabular}

TABELA 3 - Associação da inatividade física, do tabagismo e das doenças associadas com a capacidade de locomoção de indivíduos com claudicação intermitente.

* estatisticamente significante;

$\beta=$ Coeficiente de regressão.

IC $95 \%$ (intervalo de confiança de 95\%).

\begin{tabular}{lcccccc}
\hline \multirow{2}{*}{\multicolumn{1}{c}{ Fatores }} & \multicolumn{2}{c}{ Tempo de claudicação } & \multicolumn{2}{c}{ Tempo total de caminhada } \\
\cline { 2 - 7 } & $\begin{array}{l}\text { Coeficiente } \\
\text { (IC95\%) }\end{array}$ & $\mathrm{r} 2$ & $\mathrm{p}$ & $\begin{array}{c}\text { Coeficiente } \\
\beta(\mathrm{IC} 95 \%)\end{array}$ & $\mathrm{r} 2$ & $\mathrm{p}$ \\
\hline Inatividade física & $-154(-283 ;-25)$ & 0,08 & $0,02^{*}$ & $-189(-384 ;-29)$ & 0,08 & $0,02^{*}$ \\
Tabagismo & $-0,6(-153 ; 152)$ & 0,00 & 0,99 & $14(-175 ; 205)$ & 0,00 & 0,87 \\
Hipertensão arterial & $-16(-211 ; 178)$ & 0,00 & 0,86 & $107(-133 ; 348)$ & 0,01 & 0,37 \\
Diabetes melitos & $-73(-204 ; 58)$ & 0,02 & 0,26 & $-158(-321 ; 4)$ & 0,06 & 0,06 \\
Obesidade & $-81(-236 ; 73)$ & 0,02 & 0,30 & $38(-155 ; 232)$ & 0,00 & 0,69 \\
Dislipidemia & $-45(-177 ; 86)$ & 0,01 & 0,49 & $18(-151 ; 188)$ & 0,00 & 0,83 \\
Cardiopatia & $-35(-172 ; 102)$ & 0,00 & 0,61 & $-140(-308 ; 27)$ & 0,04 & 0,09 \\
Número de fatores & $-55(-102 ;-8)$ & 0,08 & $0,02^{*}$ & $-54(-113 ; 5)$ & 0,05 & 0,07 \\
\hline
\end{tabular}

280 • Rev. bras. Educ. Fís. Esporte, São Paulo, v.25, n.2, p.277-84, abr./jun. 2011 


\section{Discussão}

Os principais achados do estudo foram: 1) pacientes com DAOP apresentam elevada prevalência de comorbidades e hábitos não saudáveis; 2) a inatividade física e o número de comorbidades e/ou hábitos não saudáveis foram associados com capacidade de caminhada nesses pacientes.

A alta prevalência de comorbidades em pacientes com DAOP tem sido bem descrita na literatura (Katzel, Sorkin, Powell \& Gardner, 2001). No presente estudo, a hipertensão arterial foi a comorbidade mais prevalente nos indivíduos com DAOP, o que está em linha com outros estudos (BREVETTI et al., 2003; Garg et al., 2006; MlacaK et al., 2006; Passos, Guerra, Firmo, Vidigal \& LimaCosta, 2001). Porém, é importante destacar que comorbidades, tais como o diabetes, a obesidade, a dislipidemia e as doenças cardiovasculares, também foram frequentes na amostra do presente estudo. Dentre os hábitos não saudáveis, quase $50 \%$ dos pacientes foram considerados inativos fisicamente. Esses valores são similares aos observados em estudo prévio nacional em indivíduos com DAOP (MAKDISSE et al., 2008) e bem inferiores a outros estudos nacionais em indivíduos não doentes (PASsos et al., 2001). A menor prevalência de indivíduos inativos fisicamente entre os indivíduos com DAOP em comparação aos estudos congêneres pode ter sido causada pelo grande incentivo a prática de atividade física pelos médicos que atuam com esses pacientes e pelos diferentes métodos utilizados para quantificação dos níveis de atividade física.

A inatividade física foi associada com menor capacidade de caminhada em pacientes com DAOP. Esses resultados corroboram as evidências disponíveis na literatura que indicam a relação entre os níveis de atividade física e a capacidade de caminhada em indivíduos com DAOP. GARDNER et al. (2007) verificaram que os indivíduos com CI com menor capacidade de caminhada eram aqueles que apresentavam menores níveis de atividade física, principalmente em atividades físicas moderada e intensa. Tem sido sugerido que a relação entre a capacidade de locomoção e a inatividade física dos indivíduos com CI é decorrente da tentativa de minimizar a ocorrência dos sintomas de claudicação (GARDNER et al., 2007). Assim, para evitar os sintomas, os pacientes buscam realizar menos atividades físicas. Isso é preocupante, pois evidências de estudos longitudinais indicam que os indivíduos inativos fisicamente apresentam, em longo prazo, maior deterioração na capacidade de caminhada (GARG et al., 2006), bem como maior morbidade e mortalidade cardiovascular (Weitz, Byrne, Clagett, FarkouH, Porter, Sackett, Strandness \& Taylor Junior, 1996). Esses resultados sugerem que programas de promoção da atividade física devem ser incentivados em indivíduos com CI no sentido de melhorar as limitaçōes funcionais e o prognóstico dos pacientes.

No presente estudo, embora nenhuma comorbidade isolada tenha sido associada com a capacidade de caminhada dos indivíduos com CI, foi observada tendência de associação com algumas comorbidades, como o diabetes e as cardiopatias, o que corrobora com estudos anteriores que evidenciaram associação entre a presença de doenças crônicas e a baixa capacidade de caminhada nos indivíduos com CI (Dias, Forjaz, Cucato, Costa, Camara, Wolosker \& Marucci, 2009; Garg et al., 2006; Hasimu et al., 2006; Marubito et al., 1997; Zander, Heinke, Reindel, Kohnert, Kairies, Braun, Eckel \& Kerner, 2002). Todavia, o grande diferencial do presente estudo foi à análise do impacto das comorbidades e hábitos não saudáveis em conjunto. Esse é um aspecto relevante, pois a maioria dos estudos sobre essa temática analisou apenas fatores isolados (HoOI, Kester, Stoffers, Overdijk, van Ree \& Knottnerus, 2001), o que não reflete a característica dos pacientes atendidos em ambulatórios de cirurgia vascular. Isso é confirmado pelos resultados do nosso estudo que mostraram que 90\% dos pacientes com DAOP apresentaram duas ou mais comorbidades e/ou hábitos não saudáveis. No presente estudo, o número de comorbidades e hábitos não saudáveis foram negativamente relacionados com a capacidade de caminhada de pacientes com CI. Assim, indivíduos que apresentam maior número desses fatores são os que apresentam menor capacidade de caminhada. Esses resultados sugerem que a redução dos hábitos não saudáveis e o controle das comorbidades, poderiam melhorar a capacidade de caminhada dos indivíduos com DAOP. Todavia, essa hipótese necessita ser testada em estudos futuros com delineamento longitudinal.

Este estudo apresenta resultados que podem ser incorporados na prática diária de profissionais da área da saúde. Foi evidenciada uma associação direta entre a atividade física e a capacidade de caminhada dos indivíduos com CI. Assim, possivelmente, o estímulo à prática de atividade física poderia promover aumentos na capacidade de caminhada destes 
pacientes. Essa hipótese é corroborada por diversos estudos que confirmam a eficácia do treinamento físico na melhoria da capacidade de caminhada dos indivíduos com CI (GARDNer, MonTGOMERY, Flinn \& Katzel, 2005; Gardner \& Poehlman, 1995; Ritti-Dias, Wolosker, De Moraes Forjaz, Carvalho, Cucato, Leāo \& de Fatima Nunes Marucci, 2010). Além disso, como indivíduos com maior número de comorbidades ou hábitos não saudáveis são aqueles que apresentam menor capacidade de caminhada, parece que intervenções para controlar esses fatores poderiam promover melhoria da capacidade de caminhada de pacientes com DAOP. No entanto, o impacto do controle das comorbidades na capacidade de caminhada dos indivíduos com DAOP ainda foi pouco estudado, e os estudos disponíveis analisaram apenas os níveis de atividade física e o tabagismo. Assim, sugere-se que estudos longitudinais sejam realizados para analisar o impacto das comorbidades na capacidade de caminhada dos indivíduos com DAOP, para que seja, de fato, estabelecida uma relação de causa-efeito entre as variáveis independentes com o desfecho.

Este estudo apresenta algumas limitações que devem ser consideradas. O delineamento transversal empregado não permite estabelecer relação de causalidade. A amostra selecionada consistiu de indivíduos que estavam interessados em participar de um programa de treinamento físico, e isso, pode de alguma forma ter influenciado os resultados. As medidas de atividade física foram obtidas por método indireto, o que pode ter impactado os dados referentes aos níveis de atividade física dos indivíduos.

Como conclusão, os resultados deste estudo indicam que a inatividade física e o número de comorbidades e/ou hábitos não saudáveis estão associados com a intolerância à caminhada de indivíduos com CI.

\begin{abstract}
Association between comorbidity conditions and unhealthy habits and walking capacity in patients with intermittent claudication

Comorbid conditions and unhealthy habits, such as smoking and physical inactivity, are commonly observed in patients with intermittent claudication (IC). However, the impact of these factors on walking impairment in IC patients remains uncertain. Thus, the purpose of this study was to analyze the association between comorbid conditions and non-healthy habits and walking capacity in subjects with IC. Sixty-six men and women with IC were recruited. Subjects underwent a treadmill test in which claudication time (CT) and total walking time (TWT) were obtained. An interview was performed and comorbid conditions and unhealthy habits were obtained. Univariate linear regression was employed for statistical analysis $(p<0.05)$. The most prevalent comorbid condition was hypertension $(86.4 \%)$. Among unhealthy habits physical inactivity was the most prevalent (47.0\%). Moreover, $90.9 \%$ of the subjects had 2 or more comorbid conditions and/or unhealthy habits. Physical inactivity was associated with decreased CT ( $\beta=-154 \mathrm{~s} ; \mathrm{p}=0.02 ;$ IC95\% $=-283 ;-25 \mathrm{~s})$ and TWT $(\beta=-189 \mathrm{~s} ; \mathrm{p}=0.02 ;$ IC95\% $=-384 ;-29 \mathrm{~s})$. Furthermore, a higher number of factors were associated with lower CT ( $\beta=-55 \mathrm{~s} ; \mathrm{p}$ $=0.02 ;$ IC95\% $=-102 ;-8 \mathrm{~s}$ ). In conclusion, the results showed that physical inactivity and number of comorbid condition and/or unhealthy habits are associated to walking impairment in subjects with IC.
\end{abstract}

UnITERMS: Diseases; Risk factors; Peripheral arterial disease. 


\section{Referências}

AMERICAN DIABETES ASSOCIATION. Report of the Expert Committee on the Diagnosis and Classification of Diabetes Mellitus. Diabetes Care, Alexandria, v.20, n.7, p.1183-97, 1997.

BREVETTI, G.; SILVESTRO, A.; SCHIANO, V.; CHIARIELLO, M. Endothelial dysfunction and cardiovascular risk prediction in peripheral arterial disease: additive value of flow-mediated dilation to ankle-brachial pressure. Circulation, Hagerstown, v.108, n.2093-8, 2003.

DIAS, R.M.; FORJAZ, C.L.; CUCATO, G.G.; COSTA, L.A.; CAMARA, L.C.; WOLOSKER, N.; MARUCCI M.F.N. Obesity decreases time to claudication and delays post-exercise hemodynamic recovery in elderly peripheral arterial disease patients. Gerontology, Basel, v.55, n.1, p.21-6, 2009.

FONTAINE, R.; KIM, M.; KIENY, R. Surgical treatment of peripheral circulation disorders. Helvetica Chirurgica Acta, Basel, v.21, n.5-6, p.499-533, 1954.

GARDNER, A.W.; MONTGOMERY, P.S.; FLINN, W.R.; KATZEL, L.I. The effect of exercise intensity on the response to exercise rehabilitation in patients with intermittent claudication. Journal of Vascular Surgery, St. Louis, v.42, n.4, p.702-9, 2005. GARDNER, A.W.; MONTGOMERY, P.S.; SCOTT, K.J.; AFAQ, A.; BLEVINS, S.M. Patterns of ambulatory activity in subjects with and without intermittent claudication. Journal of Vascular Surgery, St. Louis, v.46, n.6, p.1208-14, 2007. GARDNER, A.W.; POEHLMAN, E.T. Exercise rehabilitation programs for the treatment of claudication pain: a metaanalysis. JAMA: Journal of American Medical Association, Chicago, v.274, n.12, p.975-80, 1995.

GARDNER, A.W.; SKINNER, J.S.; CANTWELL, B.W.; SMITH, L.K. Progressive vs single-stage treadmill tests for evaluation of claudication. Medicine and Science in Sports and Exercise, Madison, v.23, n.4, p.402-8, 1991.

GARG, P. K.; TIAN, L.; CRIQUI, M. H.; LIU, K.; FERRUCCI, L.; GURALNIK, J. M.; TAN, J.; McDERMOTT, M. M. Physical activity during daily life and mortality in patients with peripheral arterial disease. Circulation, Hagerstown, v.114, n.3, p.242-8, 2006. GORDON, C.C.; CHUMLEA, W.C.; ROCHE, A.F. Stature, recumbent length, and weight. Champaign: Human Kinetics, 1988. (Anthropometric Standardization Reference Manual).

HASIMU, B.; LI, J.; NAKAYAMA, T.; YU, J.; YANG, J.; LI, X.; HU, D. Ankle brachial index as a marker of atherosclerosis in Chinese patients with high casdiovascular risk. Hypertension Research, London, v.29, n.1, p.23-8, 2006.

HERMAN, S.D.; LIU, K.; TIAN, L.; GURALNIK, J.M.; FERRUCCI, L.; CRIQUI, M.H.; LIAO, Y.; McDERMOTT, M.M. Baseline lower extremity strength and subsequent decline in functional performance at 6-year follow-up in persons with lower extremity peripheral arterial disease. Journal American Geriatrics Society, Malden, v.57, n.12, p.2246-52, 2009. HIRSCH, A.T.; HASKAL, Z.J.; HERTZER, N.R.; BAKAL, C.W.; CREAGER, M.A.; HALPERIN, J.L.; HIRATZKA, L.F.; MURPHY, W.R.; OLIN, J.W.; PUSCHETT, J.B.; ROSENFIELD, K.A.; SACKS, D.; STANLEY, J.C.; TAYLOR JUNIOR, L.M.; WHITE, C.J.; WHITE, J.; WHITE, R.A.; ANTMAN, E.M.; SMITH JUNIOR, S.C.; ADAMS, C.D.; ANDERSON, J.L.; FAXON, D.P.; FUSTER, V.; GIBBONS, R.J.; HUNT, S.A.; JACOBS, A.K.; NISHIMURA, R.; ORNATO, J.P.; PAGE, R.L.; RIEGEL, B. ACC/AHA 2005 Practice guidelines for the management of patients with peripheral arterial disease (lower extremity, renal, mesenteric, and abdominal aortic): a collaborative report from the American Association for Vascular Surgery/Society for Vascular Surgery, Society for Cardiovascular Angiography and Interventions, Society for Vascular Medicine and Biology, Society of Interventional Radiology, and the ACC/AHA Task Force on Practice Guidelines (Writing Committee to Develop Guidelines for the Management of Patients With Peripheral Arterial Disease): endorsed by the American Association of Cardiovascular and Pulmonary Rehabilitation; National Heart, Lung, and Blood Institute; Society for Vascular Nursing; TransAtlantic Inter-Society Consensus; and Vascular Disease Foundation. Circulation, Hagerstown, v.113, n.11, p.e454, 2006.

HOOI, J.D.; KESTER, A.D.M.; STOFFERS, H.E.J.H.; OVERDIJK, M.M.; VAN REE, J.; W.KNOTTNERUS, J.A. Incidence of and risk factors for asymptomatic peripheral arterial occlusive disease: a longitudinal study. American Journal of Epidemiology, Cary, v.153, n.7, p.666-72, 2001.

KATZEL, L.I.; SORKIN, J.D.; POWELL, C.C.; GARDNER, A.W. Comorbidities and exercise capacity in older patients with intermittent claudication. Vascular Medicine, London, v.6, n.3, p.157-62, 2001.

KUO, H.K.;YU, Y.H. The relation of peripheral arterial disease to leg force, gait speed, and functional dependence among older adults. Journal Gerontology: a Biological Science Medicine Science, v.63, n.4, p.384-90, 2008.

MAKDISSE, M.; PEREIRA, A.D.C.; BRASIL, D.D.P.; BORGES, J.L.; MACHADO-COELHO, G.L.L.; KRIEGER, J.E.; NASCIMENTO NETO, R.M.;CHAGAS, A.C.P. Prevalência e fatores de risco associados à doença arterial periférica no projeto coraçōes do Brasil. Arquivos Brasileiros de Cardiologia, São Paulo, v.91, n.402-14, 2008.

MARUBITO, J.M.; D’AGOSTINO, R.B.; SILBERSHATZ, H.; WILSON, W.F. Intermittent claudicaion: a risk profile from the Framingham Heart Study. Circulation, Hagerstown, v.96, n.44-49, 1997. 
BARBOSA, J.P.A.S. et al.

MITCHELL, G.F. Effects of central arterial aging on the structure and function of the peripheral vasculature: implications for end-organ damage. Journal Applied Physiology, Bethesda, v.105, n.5, p.1652-60, 2008.

MLACAK, B.; BLINC, A.; POHAR, M.; STARE, J. Peripheral arterial disease and ankle-brachial pressure index as predictors of mortality in residents of Metlika County, Slovenia. Croat Medicine Journal, Zagreb, v.47, n.2, p.327-34, 2006. NORGREN, L.; HIATT, W.R.; DORMANDY, J.A.; NEHLER, M.R.; HARRIS, K.A.; FOWKES, F.G.; RUTHERFORD, R.B. Inter-society consensus for the management of peripheral arterial disease. International Angiology, Turin, v.26, n.2, p.81-157, 2007. PASSOS, V.M.A.; GUERRA, S.M.B.H.L.; FIRMO, J.O.A.; VIDIGAL, P.G.; LIMA-COSTA, M.F.F. The Bambuí Health and Aging Study (BHAS): prevalence of intermittent claudication in the aged population of the community of Bambuí and its associated factors. Arquivos Brasileiros de Cardiologia, São Paulo, v.77, n.5, p.458-62, 2001.

RITTI-DIAS, R.M.; WOLOSKER, N.; DE MORAES FORJAZ, C.L.; CARVALHO, C.R.; CUCATO, G.G.; LEAO, P. P.; DE FATIMA NUNES MARUCCI, M. Strength training increases walking tolerance in intermittent claudication patients: randomized trial. Journal of Vascular Surgery, St. Louis, v.51, n.1, p.89-95, 2010.

SOCIEDADE BRASILEIRA DE HIPERTENSÃO. SOCIEDADE BRASILEIRA DE CARDIOLOGIA. SOCIEDADE BRASILEIRA DE NEFROLOGIA. IV Diretrizes Brasileiras de Hipertensão Arterial. Arquivos Brasileiros Cardiologia, São Paulo, v.82, n.4, p.7-22, 2004.

SPRONK, S.; BOSCH, J.L.; VEEN, H.F.; DEN HOED, P.T.; HUNINK, M.G. Intermittent claudication: functional capacity and quality of life after exercise training or percutaneous transluminal angioplasty-systematic review. Radiology, Easton, v.235, n.3, p.833-42, 2005.

WEITZ, J.I.; BYRNE, J.; CLAGETT, G.P.; FARKOUH, M.E.; PORTER, J.M.; SACKETT, D.L.; STRANDNESS JUNIOR, D.E.; TAYLOR, L.M. Diagnosis and treatment of chronic arterial insufficiency of the lower extremities: a critical review. Circulation,Hagerstown, v.94, n.11, p.3026-49, 1996.

ZANDER, E.; HEINKE, P.; REINDEL, J.; KOHNERT, K.D.; KAIRIES, U.; BRAUN, J.; ECKEL, L.; KERNER, W. Peripheral arterial disease in diabetes mellitus type 1 and type 2: are there different risk factors? Vasa: Journal of Vascular Diseases, Bern, v.31, n.4, p.249-54, 2002.

ENDEREÇO

Raphael Mendes Ritti Dias

R. Arnóbio Marques, 310

50100-130 - Recife - PE - BRASIL

e-mail: raphael.dias@upe.br
Recebido para publicação: 27/ 10/ 2010

Aceito: 31/ 03/2011 